\title{
El proceso de comprometer pedidos de un paquete de productos integrado por productos del sector cerámico y productos complementarios: Parte I Descripción y caracterización de la problemática
}

\author{
$M^{\text {a }}$ M. E. ALEMANY', A. ORTIZ1', F. ALARCÓN', F. CRUZ LARIO', \\ M. A. BENGOCHEA ${ }^{2}$ \\ ${ }^{1}$ Centro de Investigación de Gestión e Ingeniería de Producción \\ Universidad Politécnica de Valencia (España) \\ ${ }^{2}$ Keraben (España)
}

\begin{abstract}
La mejora del nivel de servicio al cliente se perfila clave como un arma competitiva y de posicionamiento de las empresas en el sector cerámico. En este contexto de mejorar el nivel de servicio al cliente surge el concepto de paquete de productos (P-P) como un medio para conseguir una diferenciación basada en el diseño y la orientación al cliente. Un P-P puede definirse como una serie de productos cuya funcionalidad es complementaria y que por tanto pueden venderse de forma conjunta. Para que el valor percibido por el cliente aumente, también es necesario proporcionar una respuesta rápida y fiable a las propuestas de pedido por él realizadas. El presente artículo describe la compleja problemática del proceso de comprometer pedidos de un paquete de productos (P-P) en un dominio de cadenas de venta colaborativo extendido (Extended Collaborative Selling Chain: ECOSELL), y la compara con el proceso de comprometer pedidos tradicional. Debido a la ausencia de trabajos en este tema, se pretende establecer el punto de partida para posteriores investigaciones a través de la caracterización de todos los posibles escenarios que pueden existir en este entorno. En base a esta definición de escenarios será posible diseñar una metodología de solución genérica, capaz de abarcar todos los posibles casos, que se expondrá en un trabajo posterior.
\end{abstract}

Palabras clave: Proceso de Comprometer Pedidos, Paquete de Productos, Sector Cerámico, Productos Complementarios.

The order promising process of a product pack composed by products belonging to the tile sector and complementary products: Part I Problem description and characterisation.

The improvement of the customer service level is a key aspect in the competitive positioning of the ceramic sector companies. Within this context of improving the customer service level, the concept of "Product Pack" (P-P) arises as a mean to achieve product differentiation based on integral design and customer orientation. A P-P can be defined as a group of products that are complementary in its function and can be commercialised jointly. In order to increase the value perceived by the customer, it is necessary as well to give a rapid and reliable response to his/her order proposals. This article presents the problem description of the order promising process of a P-P in an Extended Collaborative Selling Chain (ECOSELL) domain, and develops a comparison with the traditional order promising process. Due to the absence of research about this topic, it is the objective of this article to define the starting point for future research by the characterization of all the possible scenarios that may appear in this context. On the basis of the aforementioned definition it is possible to design as well a generic methodology able to solve all the possible cases, to be exposed in a further work.

Keywords: Order Promising Process, Product Pack, Tile Sector, Complementary Products.

\section{INTRODUCCIÓN}

Las nuevas presiones del mercado global hacen necesario potenciar las actividades del final de la cadena de valor del sector cerámico, especialmente las relacionadas con el objetivo de maximizar la satisfacción del cliente. Para ello es necesario, entre otras cosas, una respuesta rápida y fiable a las peticiones por él realizadas. Estrechamente vinculado a lo anterior se encuentra el concepto de "comprometer pedidos". La mayoría de los trabajos consultados utilizan el término "proceso de comprometer pedidos" para referirse al conjunto de actividades que se ponen en marcha con el objetivo de dar respuesta a una determinada propuesta de pedido por parte de un cliente. Dicha respuesta puede significar la aceptación o rechazo de una propuesta de pedido de un cliente. En caso de existir una aceptación, el punto de venta adquiere un compromiso en cuanto a cantidades y fechas de entrega con el cliente que debe cumplir a través de una utilización eficiente de los recursos de la red. La realización correcta del anterior proceso no sólo impacta sobre el nivel de satisfacción del 
cliente sino también sobre los costes de la red asociados a la utilización de los recursos disponibles.

En los últimos años, las investigaciones sobre el proceso de comprometer pedidos se han incrementado sustancialmente. Sin embargo, los trabajos revisados se centran en propuestas de pedidos compuestas por un único producto. El presente artículo cubre el caso de propuestas de pedido de paquetes de productos (P-P). Un P-P puede definirse como una serie de productos cuya funcionalidad es complementaria y que por tanto pueden venderse de forma conjunta. Estos productos, en el caso más general, pueden pertenecer a diferentes cadenas de suministro, que compondrían lo que en adelante se denominará red.

El sector cerámico no es ajeno a esta necesidad de satisfacer al cliente, muy al contrario, la competencia se incrementa día a día, en parte como consecuencia del gran aumento de la producción de países emergentes como China, Brasil y países de la zona Asia-Pacífico (1). Este incremento en la satisfacción al cliente depende de muchos aspectos como la entrega a tiempo y en las condiciones adecuadas del producto seleccionado por el cliente, pero también de ser capaces de ofrecerle al cliente un producto cada vez más atractivo y completo. Optar por la diferenciación es la clave del sector en Europa, por lo que se debe apostar por las actividades de comercialización en general y diseño realizándolo todo de forma integrada (2). Uno de los campos de futura investigación relacionados con el diseño es el desarrollo de productos con nuevas propiedades y funciones (3). En este sentido, algunas de las tendencias de diseño que empiezan a emerger en el sector es el de ofrecer ambientes o P-P, es decir, un conjunto de productos combinados (pavimento, revestimiento, muebles de cocina, grifería, electrodomésticos, etc.). Estos ambientes presentan varias ventajas importantes; en la fase de diseño todas las empresas involucradas pueden diseñar el ambiente de forma más atractiva y rentable, la logística combinada puede representar ventajas, etc. Desde el punto de vista del cliente las ventajas también son evidentes; además de un ambiente atractivo, se unifica en un único punto de venta todo el proceso de compra. De esta manera, el cliente no debe visitar distintos puntos de venta para comprar todos los elementos del ambiente ya que en un solo punto se gestionan pagos y logística de entrega. Las ventajas competitivas son evidentes, pero como siempre, para que resulten rentables hay que gestionar el proceso de forma adecuada, ya que la complejidad aumenta debido a la necesidad de coordinar de forma adecuada diversas cadenas de suministro.

En el presente artículo se plantea la forma de caracterizar adecuadamente el proceso de comprometer pedidos para el caso de ambientes o P-P. De esta forma, se establece un primer paso para gestionar adecuadamente este proceso y, por tanto, se desarrolla una de las partes básicas del escenario descrito anteriormente.

El artículo se encuentra estructurado de la siguiente manera: en primer lugar se lleva a cabo una revisión bibliográfica en torno al tema. Posteriormente se realiza una descripción de la problemática del proceso tradicional de comprometer pedidos para establecer las diferencias con respecto al caso de un P-P. A continuación, se exponen las características de la red involucrada en la elaboración de un P-P compuesto por productos del sector cerámico y productos complementarios así como las particularidades de la propia propuesta de pedido. En base a lo anterior se describen los diferentes escenarios que se pueden presentar para, finalmente, extraer una serie de conclusiones.

\section{REVISIÓN BIBLIOGRÁFICA}

Durante la tarea de comprometer pedidos se debe decidir sobre la aceptación o no de éstos y el establecimiento de las fechas de entrega prometidas a los clientes. Esta tarea requiere comprobar si existe la suficiente disponibilidad de productos finales, materiales y/o componentes, capacidad de los recursos de fabricación y de distribución para satisfacer los requerimientos del cliente. Con el objetivo de facilitar este trabajo se han definido varios conceptos: "disponible a prometer" (ATP: Available-to-Promise), "capacidad disponible a prometer" (CTP: Capable-to-Promise) y "capacidad de distribución disponible a prometer" (DTP: Deliver-to-Promise). La revisión de la literatura realizada sobre este tema muestra que no existe un consenso a la hora de definir los anteriores conceptos. Algunas definiciones de estos términos pueden encontrarse en ((4), (5), (6), (7) , (8) y (9)).

Recientemente se han publicado algunos trabajos que pueden ser de ayuda para clarificar la anterior confusión. Estos trabajos proponen un marco de clasificación para la literatura relacionada con el proceso de comprometer pedidos, o en ocasiones equivalente ATP o CTP. (7) distinguen entre modelos ATP "push" y "pull". (10) proponen diferentes dimensiones para clasificar la bibliografía existente. (11) propone una interesante clasificación de los sistemas ATP avanzados (AATP) basados en tres características: el "nivel de disponibilidad" que se comprueba (inventario de productos finales o recursos de la cadena de suministro), el "modo de operación" (en tiempo real o no) y la "interacción con la planificación de los recursos de fabricación" (activa o pasiva).

Normalmente, en los trabajos consultados, el "nivel de disponibilidad" que se comprueba es el relativo al inventario de productos finales (ATP), siendo más escasos los trabajos centrados en el cálculo de la disponibilidad de capacidad los recursos de fabricación (CTP) y no teniendo conocimiento de ningún trabajo que compruebe la disponibilidad de capacidad de los recursos de distribución (DTP). Tampoco se tiene constancia de investigaciones que comprueben la disponibilidad de recursos involucrados en servicios asociados a un cierto producto como, por ejemplo, los instaladores.

Son numerosos los trabajos que tratan con propuestas de pedido con un valor específico para la fecha de entrega y la cantidad de un producto, pero muy pocos proponen escenarios en los que se trate el precio ((12), (13)), o la posibilidad de utilizar productos sustitutivos ((11), (14), (15)). Además generalmente se contempla un único valor para las anteriores características de la propuesta de pedido (fecha, lugar, cantidad, etc.). Sin embargo, en la realidad el cliente puede proponer un conjunto de posibles fechas de entrega ((14), (15), (16)). Además dicho conjunto de valores puede tener asociada una función de satisfacción por parte del cliente o de la red (16).

El creciente número de artículos recientes en torno al tema del proceso de comprometer pedidos, muestra que se trata de un área de investigación en expansión ((11), (13), (14), (15), (17), (18), (19), (20), (21), (22)). Sin embargo, toda la investigación se centra en una única empresa o cadena de suministro. No se tiene conocimiento acerca de ningún trabajo relacionado con el complejo proceso de comprometer pedidos de un P-P en el que varias cadenas de suministro se ven implicadas. El presente artículo pretende describir la problemática para este tipo de pedidos, estableciendo claramente las diferencias y dificultades añadidas con respecto al proceso tradicional de comprometer pedidos. 


\section{EL PROCESO DE COMPROMETER PEDIDOS}

Como se ha expuesto en el apartado anterior, no existe un consenso a la hora de definir la terminología relacionada con el proceso de comprometer pedidos. A continuación se presentan una serie de definiciones clave en el marco de la presente investigación, que han sido desarrolladas por lo autores a partir de la revisión bibliográfica:

- ATP: El ATP (disponible a prometer) es la cantidad disponible, real o planificada, de producto final no comprometida con ningún cliente. Esta cantidad no comprometida se deriva del inventario disponible, los suministros esperados y los pedidos comprometidos de los clientes.

- CTP: El CTP (capacidad disponible a prometer) es la capacidad disponible, real o planificada, no comprometida de aquellos recursos implicados en la fabricación del producto perteneciente a un pedido de un cliente. Estos recursos no sólo incluyen los recursos de fabricación sino también las materias primas, los materiales y los componentes necesarios para satisfacer el pedido.

- DTP: El DTP (capacidad de distribución disponible a prometer) es la capacidad disponible, real o planificada, no comprometida de los recursos de distribución (almacenamiento y transporte).

En general, la forma en la que se utilizan las anteriores disponibilidades a la hora de comprometer un pedido con un cliente es la siguiente (figura 1): el proceso de comprometer pedidos comienza con la comparación entre la cantidad de un determinado producto demandada por el cliente para una fecha y la cantidad disponible del mismo no comprometida con ningún cliente (ATP) hasta la misma fecha. Si la cantidad ATP es suficiente, se adquiere un compromiso de entrega con el cliente. En caso de que dichas cantidades sean insuficientes, sería posible comprometer pedidos en base también a las cantidades CTP, que muestran la capacidad sobrante teniendo en cuenta los pedidos comprometidos y los suministros esperados (23). Pero que exista suficiente ATP y/o CTP para servir un pedido no asegura su entrega a tiempo. Para ello, habrá que comprobar si existe la suficiente capacidad de distribución no comprometida (DTP) para transportar la cantidad requerida desde los lugares de aprovisionamiento hasta el lugar de entrega.

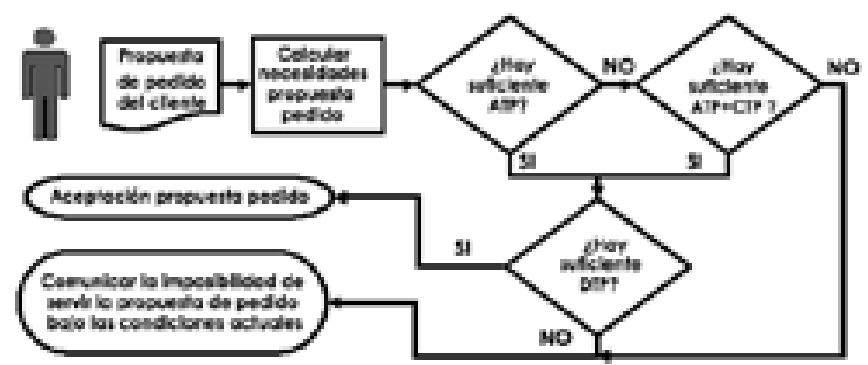

Fig. 1- Proceso genérico de comprometer pedidos.

\section{DESCRIPCIÓN DE LA PROBLEMÁTICA DEL PROCESO DE COMPROMETER PEDIDOS DE P-P EN UN ENTORNO ECOSELL}

En el caso más general, cada uno de los productos integrantes del P-P puede ser proporcionado por diferentes cadenas de suministro, en cuyo caso, a la hora de comprometer el pedido será necesaria la coordinación de todas ellas. Con el objetivo de dar respuesta a esta situación, se ha desarrollado un módulo denominado COM (Collaborative Order Management) en el marco del Proyecto Europeo (ECOSELL). En el contexto ECOSELL, varias cadenas de suministro colaboran para proporcionar una proposición de valor al cliente superior a la que cada una de ellas puede alcanzar por separado.

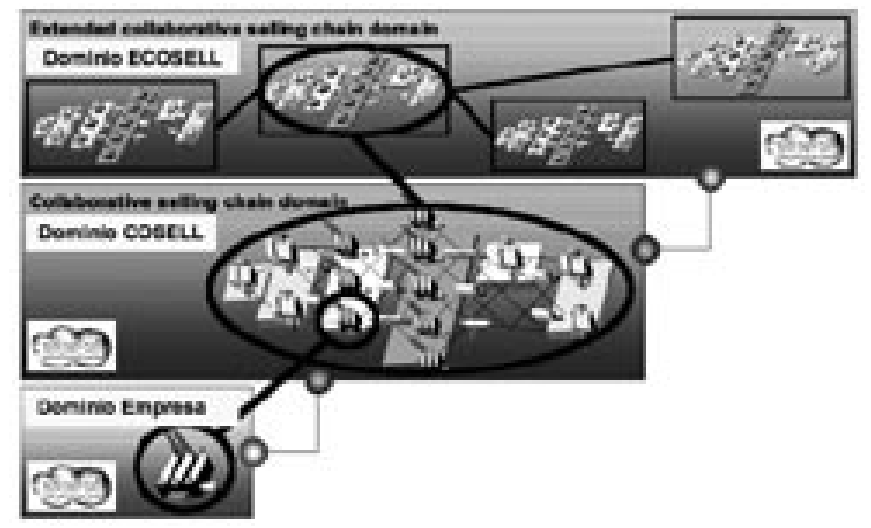

Fig. 2- Dominio ECOSELL

El módulo COM propuesto ha sido aplicado a dos pilotos, ambos integrados por dos cadenas de suministro. El piloto 1 está compuesto por cadenas de suministro pertenecientes al sector cerámico y de producto complementario y el piloto 2 , por cadenas de suministro pertenecientes a los sectores de los neumáticos y de los aparatos de audio para coches. En el presente trabajo nos centraremos en el piloto 1 . Así, ante la demanda de un determinado ambiente o P-P compuesto por productos del sector cerámico y productos complementarios (como p.e. un "ambiente cocina", "ambiente cuarto de baño", etc.) por parte de un cliente, existen dos posibilidades: que el cliente elija cada producto del ambiente independientemente, o bien presentarle una gama de "ambientes" completos apareciendo cada uno de ellos como un producto único (P-P). Con la segunda opción, se incrementa el valor del producto proporcionado al cliente. Pero esta alternativa tiene implicaciones importantes no sólo a nivel estratégico y de diseño del producto, sino también a nivel del proceso de comprometer este tipo de pedidos. Con el módulo COM de ECOSELL se pretende llevar a cabo este proceso de una manera coordinada:

- Trabajar sin COM implicaría proporcionar una fecha de entrega predefinida, bien basada en la experiencia del vendedor, bien basada en tiempos de suministro fijados de antemano. El punto de venta enviaría una propuesta de pedido individual a cada cadena de suministro para confirmar el pedido del P-P. Sin embargo, con este procedimiento cabe esperar los siguientes resultados:

o Ineficiente coordinación y utilización de los recursos de las cadenas de suministro

o Elevado tiempo de respuesta percibido por el cliente

o Se incrementa la posibilidad de entregas tardías o descoordinadas

- Para evitar estos resultados surge el módulo COM de ECOSELL. Con COM el punto de venta enviaría la propuesta de pedido a un módulo central de gestión que tendría en cuenta las disponibilidades de todas las cadenas de suministro y establecería de manera coordinada la posibilidad o no de cumplir con los requisitos del cliente de una manera eficiente.

Por tanto, el problema a abordar por el módulo COM 
puede resumirse de la siguiente manera: dada una propuesta de pedido de un P-P por parte de un cliente, el sistema debe dar una respuesta en tiempo real a lo solicitado por el cliente en base a los compromisos existentes con los clientes, el inventario de productos finales y los suministros planificados en las múltiples cadenas de suministro (ATP), las disponibilidades de capacidad de fabricación y materiales no comprometidas (CTP), la disponibilidad de capacidad de los proveedores de servicios no comprometida (CTP) y las disponibilidades de capacidad de distribución no comprometidas (DTP).

El proceso de comprometer pedidos de P-P presenta una mayor complejidad en comparación con el proceso de comprometer pedidos tradicional por varias razones. En primer lugar, es necesario coordinar cadenas de suministro normalmente con diferentes objetivos, políticas y prioridades a la hora de atender a sus clientes. Por otro lado, cada cadena de suministro puede presentar diferentes estrategias de fabricación, con lo que será necesario combinar el cálculo de diferentes "niveles de disponibilidad". Finalmente, la propia propuesta de pedido de un P-P permite definir diferentes fechas de entrega y lugares de entrega para cada uno de los productos del P-P, pudiendo existir algún tipo de relación entre ellos que, obviamente, debe respetarse.

De lo dicho anteriormente se deduce que, en el proceso de comprometer pedidos de P-P impactan de manera importante tanto las características de la red involucrada en la obtención del P-P, como las características de dicho pedido.

En el presente artículo el ambiente o P-P elegido está compuesto por productos del sector cerámico y productos complementarios. La red involucrada en la obtención de este P-P se encuentra integrada por una cadena de suministro perteneciente al sector cerámico y otra de productos complementarios. A continuación se detallan las características de la red y del pedido elegido.

\section{CARACTERÍSTICAS DE LA RED INTEGRADA POR CADENAS DE SUMINISTRO PERTENECIENTES AL SECTOR CERÁMICO Y DE PRODUCTO COMPLEMENTARIO}

Dentro del ámbito del Proyecto Europeo ECOSELL, se considera que el "Centro del Universo" más adecuado de una cadena de suministro es el Ensamblador u OEM (Original Equipment Manufacturer). Por tanto, el presente estudio se centra en parte de cada cadena de suministro: desde el OEM hasta el consumidor final (aguas abajo del OEM). En caso del piloto 1 los OEMs de ambas cadenas de suministro coinciden con los fabricantes de azulejos y revestimientos cerámicos, por un lado, y los fabricantes de producto complementario, por otro.

Cada cadena de suministro comprende, entre otros, OEMs, distribuidores, minoristas, mayoristas, intermediarios y clientes finales. Además, en el caso de redes de suministro que proporcionan un P-P se hace imprescindible considerar un concepto adicional: el Punto de Consolidación (Consolidation Point: $\mathrm{CP}$ ). El CP es el lugar donde coinciden físicamente y temporalmente todos o algunos de los productos del P-P y el lugar a partir del cual estos productos se manipulan conjuntamente como si se tratasen de un único producto. El CP puede ser la casa del cliente o un Centro de Consolidación Logístico perteneciente a la red. En algunas ocasiones, las propias características del pedido definen el punto de consolidación, pero en otras, la red tiene la posibilidad de establecer un lugar o nodo que actúe como centro de consolidación. En este último caso, la adecuada asignación del mismo puede incrementar considerablemente la eficiencia de la red.

En el caso del piloto 1 la red se encuentra integrada por dos cadenas de suministro (figura 3). La cadena de suministro del sector cerámico objeto de este estudio forma parte de un grupo dedicado a la fabricación y comercialización de pavimento y revestimiento cerámico. La elaboración de estos productos se lleva a cabo por varias plantas bajo una estrategia de fabricación contra almacén. Sin embargo, en la industria del pavimento y revestimiento cerámico cada modelo debe ser almacenado por subgrupos en función del tono y del calibre, lo que supone la necesidad de un sistema de almacenamiento y distribución que tenga en cuenta este hecho (4) Este hecho característico del sector cerámico debe ser considerado en el proceso de comprometer pedidos y, especialmente, en el cálculo del ATP. Adicionalmente a los almacenes existentes en cada planta de fabricación, el modelo actual presenta un almacén central con picking y diversos almacenes asociados a cada punto de venta. Las entregas desde el almacén central pueden realizarse pasando por el punto de venta o directamente al cliente. Además, el cliente tiene la opción de contratar o no el servicio de instalación.

Por su parte, la cadena de suministro de producto complementario se encuentra formada también por varias fábricas que funcionan bajo pedido. Esta cadena de suministro posee almacenes locales o puntos de tránsito para optimizar la ruta de vehículos, además de diversos puntos de venta. En este caso la propuesta de pedido del cliente, normalmente, incluye el servicio de instalación. La red tiene la posibilidad de definir como CP cualquier nodo de almacenaje de la red salvo las plantas de producción.

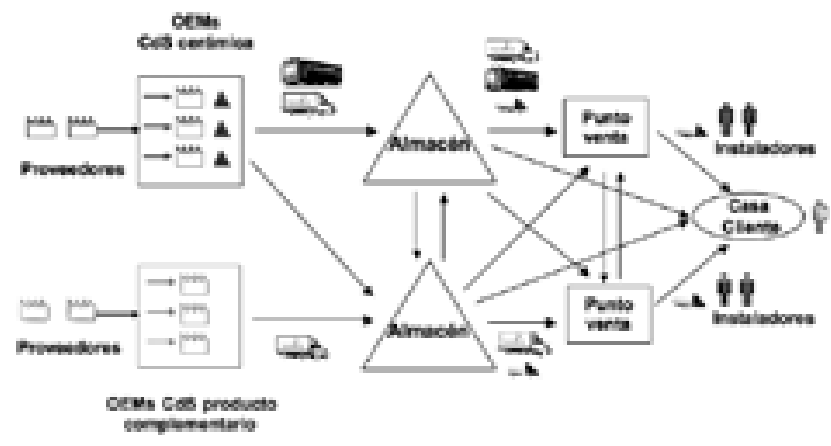

Fig. 3- Red responsable de la fabricación del ambiente o P-P integrado por productos del sector cerámico y productos complementarios

\section{CARACTERÍSTICAS DEL PEDIDO DE UN P-P COMPUESTO POR PRODUCTOS DEL SECTOR CERÁMICO Y PRODUCTOS COMPLEMENTARIOS}

La red de suministro objeto de estudio ofrece al cliente la posibilidad de personalizar los siguientes parámetros de la propuesta de pedido (25):

- Descripción del P-P, incluyendo la descripción y/o el modelo de cada componente o producto del P-P.

- Cantidad del P-P, especificando la cantidad de cada uno de los productos que integran el P-P.

- Fecha de entrega: bien para el P-P o bien para cada 
uno de sus componentes por separado. En el caso de que se soliciten diferentes fechas de entrega para cada uno de los componentes del P-P, éstas pueden tener algún tipo de relación (por ejemplo, el producto A se debe entregar 5 días después del producto B).

- Lugar de la entrega: bien para el P-P o bien para cada uno de sus componentes por separado. En el caso de que se soliciten diferentes lugares de entrega para cada uno de los componentes del P-P, éstos pueden tener algún tipo de relación (por ejemplo, el producto A se debe entregar en un radio de 50 $\mathrm{kms}$. respecto del lugar de entrega del producto $B$ ).

- Otros factores: el cliente puede o no elegir si desea la instalación del P-P o de sus componentes por separado, dependiendo de las características de cada cadena de suministro. Asimismo, cuando el cliente conozca los precios de venta del producto que desea adquirir, o cuando quiera especificar un límite al precio que estaría dispuesto a pagar, podría reflejar dicho precio en la propuesta de pedido.

La personalización de estos parámetros se realizaría, para el caso de una propuesta de pedido de un solo producto, en base a dos dimensiones: Una primera dimensión caracterizada por quién propone el valor del parámetro en cuestión, y una segunda dimensión caracterizada por el tipo de valor que se propone. Adicionalmente, para el caso de propuestas de pedidos de P-P aparece una tercera dimensión que trata sobre la existencia de posibles aspectos de dependencia entre los productos integrantes en la propuesta de pedido. En cuanto a las tres dimensiones consideradas en este apartado se admiten las siguientes posibilidades (figura 4):

- ¿Quién propone el valor?. La situación considerada más normal y probable en los sectores citados es que el cliente proponga una fecha de entrega o solicite de la red una respuesta en este sentido. Por ello, se admiten todos los casos posibles (elige la empresa libremente, elige la empresa condicionada por el cliente, elige el cliente libremente o elige el cliente condicionado por la empresa) con respecto a los campos fecha y lugar de entrega.

- ¿Qué tipo de valor?. Se admite que, para los campos fecha y lugar de entrega, el cliente pueda proponer tanto un valor único, como un conjunto de valores. En el caso de que el cliente incluya en su propuesta un conjunto de valores puede, además, especificar una función de satisfacción asociada a los valores de dicho conjunto. Un caso particular de un conjunto de valores con función de satisfacción asociada para el parámetro o campo "fecha de entrega" podría ser, por ejemplo, "el mes que viene pero preferiblemente a principios de mes". Análogamente, se podría dar el caso de que el cliente solicite la entrega de su producto "lo más cerca posible de...", en referencia al campo "lugar de entrega".

- ¿Existen dependencias?. Se admite, por parte del cliente, la vinculación de los campos fecha y lugar de entrega de un componente de un paquete de productos con otro componente, existiendo, por tanto, dependencia. También se puede vincular la fecha y/o lugar de entrega de un componente a la fecha y/o lugar de entrega de otro componente que esté a su vez vinculado con un tercero, y así sucesivamente, pudiendo establecerse, de esta forma, varios niveles de dependencia. Cuando existe una dependencia, existen componentes dependientes y otros independientes. Se admiten múltiples niveles de dependencia, siempre y cuando estén correctamente definidos (es decir, que no sean dependencias o referencias circulares) y exista, al menos, un componente independiente.

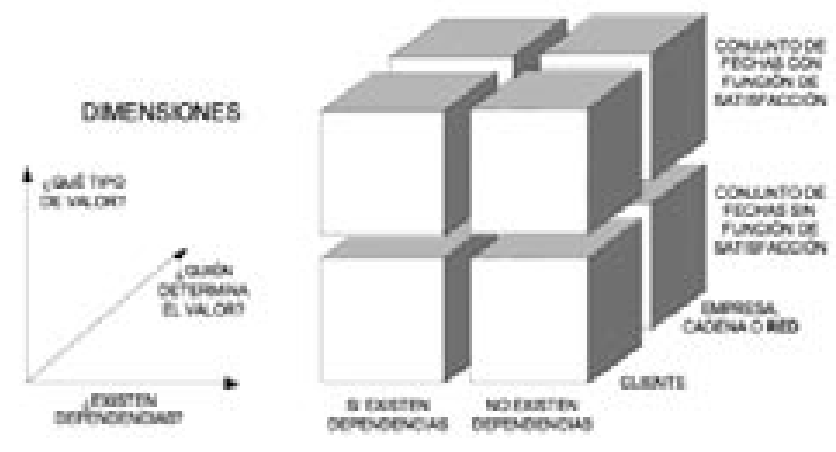

Fig. 4- Dimensiones para la personalización de los parámetros de una propuesta de pedido de un P-P genérica

\section{POSIBLES ESCENARIOS}

Una vez expuestas las características de la red y de la propuesta de pedido para el piloto 1 , se pasa a describir las diferentes situaciones que se pueden presentar (definición de escenarios) cuando llega una propuesta de pedido de un P-P a la red. Este es, sin duda, el punto de partida para diseñar una metodología de solución que sea capaz de manejar cualquier escenario que se pueda plantear. Con el objetivo de ganar en claridad a la hora de exponer los posibles escenarios de una propuesta de pedido de un P-P y al mismo tiempo remarcar las diferencias existentes con respecto al proceso de comprometer pedidos tradicional, en primer lugar se presentan los posibles escenarios en el caso de propuestas de pedidos de un solo producto y en segundo lugar los correspondientes a un P-P.

Es necesario recalcar que los escenarios se definen en base a aquellos parámetros personalizables del pedido y aquellas dimensiones que tienen un impacto en el procedimiento de solución que se propondrá en un trabajo posterior (26). Por esa razón, los escenarios se plantean con respecto a los parámetros lugar de entrega y fecha de entrega, y las dimensiones: “¿quién propone el valor?" y “¿existen dependencias?". Con respecto a la dimensión "¿qué tipo de valor?", la metodología de solución propuesta (26) considera que un valor único es una particularización de un conjunto de valores, por lo que ésta se encuentra diseñada para trabajar siempre con un conjunto de valores que serán ordenados en base a una función de satisfacción bien definida por el cliente o bien por la red.

\subsection{Posibles escenarios para una propuesta de pedido tradicional}

Como se ha comentado anteriormente, la tercera dimensión “¿existen dependencias?" no tiene sentido en el caso de una propuesta de pedido tradicional. Por tanto, en base a la dimensión “¿quién propone el valor?" sólo pueden presentarse dos escenarios:

- ESCENARIO 1) El cliente propone un conjunto de valores para la fecha y lugar de entrega. La red deberá comprobar si es posible o no satisfacer los requerimientos del cliente.

- ESCENARIO 2) La red propone un conjunto de valores para la fecha y lugar de entrega. En este caso, el cliente no propone unos requerimientos específicos con respecto a la fecha y lugar de entrega, siendo la red la que debe proponerlos. y el cliente proceder a su aceptación final. Este sería el caso, por ejemplo, de servir tan pronto como sea posible. 


\subsection{Posibles escenarios para pedidos de un P-P}

En el caso de una propuesta de pedido de un P-P la situación es más compleja porque al existir varios productos los parámetros personalizables con respecto a la fecha de entrega y el lugar de entrega pueden ser diferentes con respecto a alguna o todas las dimensiones. Así, los productos integrantes del P-P pueden presentar diferentes fechas y/o lugares de entrega (por ejemplo: los azulejos deben entregarse en 15 días y los productos complementarios en 20 días). Además pueden existir relaciones entre las diferentes fechas y/o lugares de entrega (por ejemplo, los productos complementarios deben entregarse entre 2 y 4 días después de los azulejos con el objetivo de permitir la instalación de estos últimos). a los que debe hacer frente una metodología de solución propuesta son los que aparecen en la figura 5 .

En el caso más general, el cliente puede proponer un conjunto de valores para la fecha y/o lugar de entrega de todos los productos del P-P, o bien éstos pueden ser propuestos por la red, o bien parte pueden ser propuestos por el cliente y parte por la red. En el primer caso, todos los productos del P-P pueden presentar la misma fecha de entrega y/o lugar de entrega o, por el contrario, algunos de ellos presentar diferentes valores de estos atributos. Para aquellos con valores diferentes, puede existir o no una relación o función de dependencia entre ellos. En el segundo caso, es la red la que propone rangos de valores para las fechas $\mathrm{y} / \mathrm{o}$ lugares de entrega de todos los productos del P-P. De nuevo,

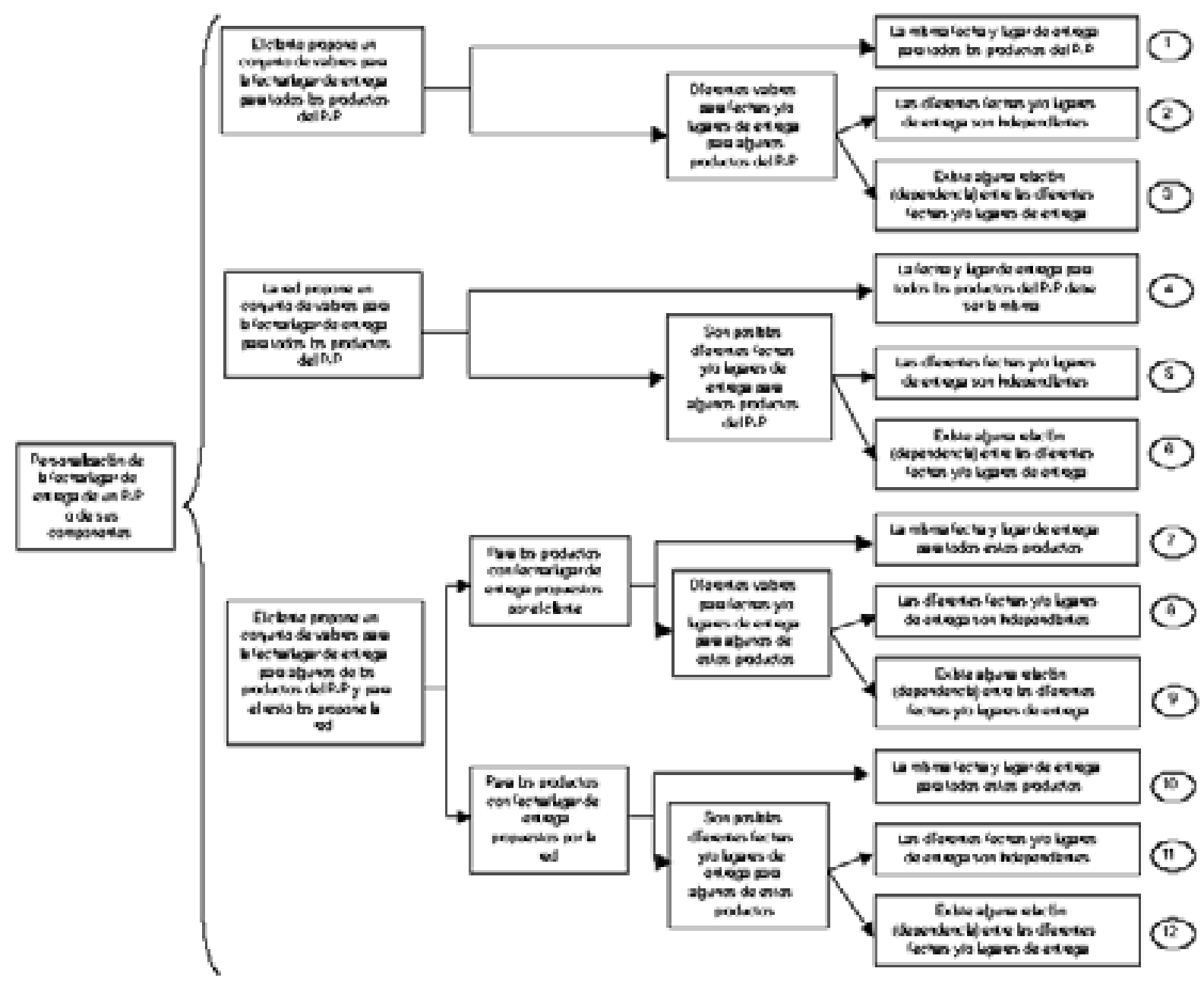

Fig. 5- Definición de escenarios para el proceso de comprometer pedidos de un P-P

Para este caso, se puede considerar que existen dos niveles de dependencia: los azulejos constituirían los productos independientes (nivel 1) y los productos complementarios los dependientes (nivel 2) puesto que su fecha de entrega depende de la fecha de entrega de los azulejos. Pero, la situación puede ser incluso más complicada en el caso de que el P-P esté compuesto por más de dos productos pudiendo existir más de dos niveles de dependencia.

Por tanto, en base a los parámetros personalizables lugar de entrega y fecha de entrega para cada uno de los productos del P-P y con respecto a las dimensiones ¿quién propone el valor?" y “¿existen dependencias?" los posibles escenarios el valor de los dos atributos anteriores puede ser idéntico para todos los productos o existir algunos con atributos diferentes. Para aquellos que sean diferentes puede existir una relación de dependencia o no. Finalmente, es posible que se presente una situación mixta: el cliente puede proponer valores para alguno de los productos del P-P y la red para el resto, repitiéndose de nuevo el esquema de dependencia descrito anteriormente. Para clarificar el árbol de posibles escenarios, en la figura 6 aparecen algunos de los escenarios más representativos en el caso objeto de estudio. Hay que resaltar que cuando existen algunos productos cuya fecha y/o lugar de entrega es propuesto por el cliente y otros por la red, para clasificar 


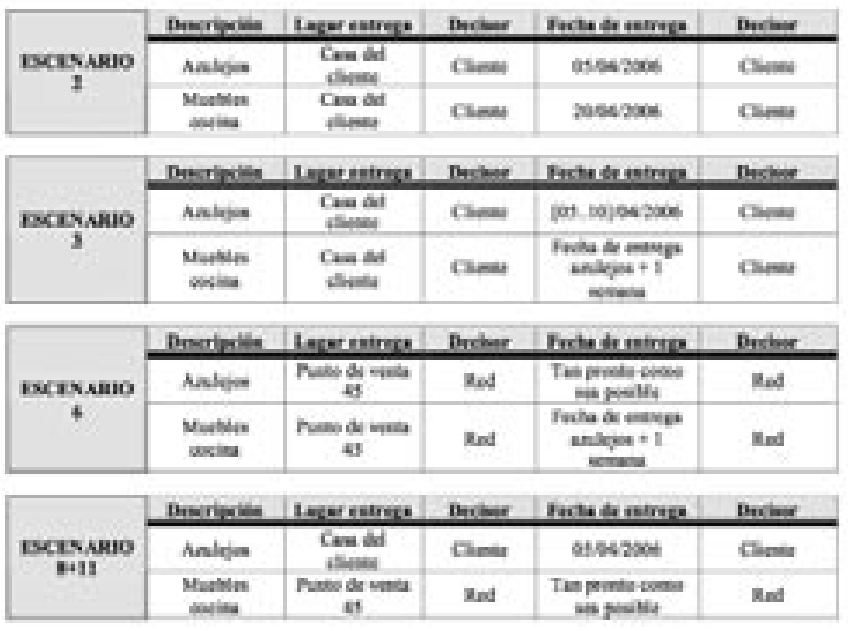

Fig. 6- Algunos ejemplos de escenarios del ambiente o P-P integrado por productos del sector cerámico y productos complementarios

el caso dentro de los posibles escenarios, debe definirse una combinación de (7 ó 8 ó 9) más (10 ó 11 ó 12).

\section{CONCLUSIONES}

La producción en el ámbito global del azulejo cerámico hace necesaria la revisión de aquellos paradigmas azulejeros enfocados en la mayor parte a la producción. Se requiere potenciar todosloselementosque conduzcan ala diferenciación, especialmente los relacionados con la comercialización y el diseño. La mejora de ambos elementos repercute directamente en el nivel de satisfacción del cliente. Íntimamente relacionado con la comercialización y la satisfacción del cliente se encuentra el proceso de comprometer pedidos. En el presente artículo se ha mostrado el gran impacto que tiene el proceso de comprometer pedidos sobre el nivel de satisfacción del cliente y la utilización eficiente de los recursos de las empresas.. Desde el punto de vista del diseño, el entorno dinámico y competitivo en el que nos encontramos hace que las empresas busquen nuevas formas de incrementar el valor percibido por sus clientes. Con este objetivo surge el concepto de P-P con importantes implicaciones de colaboración no sólo en el diseño del producto sino también en el proceso de comprometer pedidos. Trabajar con un P-P complica sustancialmente el proceso de comprometer pedidos: por un lado es necesario llevar a cabo diferentes comprobaciones acerca del nivel de disponibilidad (ATP, CTP y DTP) y coordinar múltiples cadenas de suministro con diferentes características (estrategias de fabricación, políticas para servir pedidos, etc.). Por otro lado, las propias características de la propuesta de pedido pueden hacer que los productos integrantes del P-P presenten valores diferentes con respecto a las fechas y lugares de entrega, pudiendo presentarse algún tipo de relación entre ellos que debe respetarse. Como paso previo a la propuesta de una metodología de solución para abordar el anterior problema, se ha procedido a la definición de los posibles escenarios que pueden presentarse. Esta definición de escenarios es el punto de partida para describir una propuesta de metodología de solución a la vez que presenta un marco de referencia para las empresas que actualmente o en el futuro se estén planteando el uso del concepto de P-P, ya que permite visualizar todas las posibles alternativas que se pueden dar relacionadas con el proceso de comprometer pedidos. Las distintas cadenas de suministro deben de evaluar su capacidad para abordar y ofrecer estos escenarios o no. Una vez evaluada esta posibilidad, si se desea implementarla, es necesario utilizar la metodología adecuada. Esta metodología se presenta en un trabajo posterior (26).

\section{AGRADECIMIENTOS}

Esta investigación ha sido desarrollada parcialmente en el marco del Proyecto 'Extended Collaborative Selling Chain' (ECOSELL), GRD1-2001-40692, perteneciente al V Programa Marco de la Unión Europea (Competitive and Sustainable Growth).

\section{BIBLIOGRAFÍA}

1. E. Sánchez, J. García-Ten, M. Regueiro. «Materias para la industria cerámica española. Situación actual y perspectivas». Bol. Soc. Esp. Ceram. V., 45 [1] 1-12, (2006)

2. J. Albors, J.L. Hervás, «La industria cerámica europea en el siglo XXI. Retos tecnológicos y desafíos de la próxima década». Bol. Soc. Esp. Ceram. V., 45 [1], 13-21, (2006).

3. A. Barba. «From Chemical Engineering to Ceramic Technology: A Review of Research at the Instituto de Tecnología Cerámica». Bol. Soc. Esp. Ceram. V., 44 [3], 155-168 (2005).

4. B. Fleischmann, H. Meyr. «Planning Hierarchy, Modelling and Advanced Planning Systems». In Handbooks in Oper Res Manag Sci, 11,pp. 457-523 (2003).

5. APICS, American Production and Inventory Control Society., J.F. Cox, J.H. Blackstone, M.S. Spencer, pp. 161 y 68.1994.

6. C. Svensson, K. Steger-Jensen. «Creating synergy through the integration of advanced planning systems and configuration». Collaborative systems for production management, Jagdev, Wortmann y Pels (IFIP) (2003)

7. M.O. Ball, C.-Y. Chen, Z.-Y. Zhao «Available to promise», Handbook of Quantitative Supply Chain Analysis: Modeling in the E-Business Era. Editado por D. Simchi-Levi, S.D. Wu, Z.M. Shen.(Kluwer Academic Publishers), pp. 447-483 (2004).

8. G. Berning, M. Brandenburg, K. Gürsoy, J.S. Kussi, V. Mehta, F.-J. Tölle. «Integrating collaborative planning and supply chain optimization for the chemical process industry (I)-methodology». Comput Chem Eng, 28, 6-7, pp. 913-927 (2004).

9. F. Alarcón, M.M.E. Alemany, A. Ortiz, F.C. Lario, "“Order promising” y conceptos asociados: revisión de significados e interpretaciones y propuesta para su clasificación», IX Congreso de Ingeniería de Organización, Gijón, 8 y 9 de septiembre de (2005).

10. P. Keskinocak, S. Tayur. «Due Date management policies». Handbook of Quantitative Supply Chain Analysis: Modeling in the E-business Era, edited by Simchi-Levi, D., Wu, S. D. and Shen, Z.M., pp. 485-554 (2004).

11. R. Pibernik, «Advanced available-to-promise: Classification, selected methods and requirements for operations and inventory management». Int J Prod Econ, 93-94, pp. 239-252 (2005).

12. B. Jeong, S.-B. Sim, H.-S. Jeong, S.-W. Kim «An available-to-promise sytem for TFT LCD manufacturing in supply chain» Comput Ind Eng, 43, pp. 191-212 (2002).

13. F.T.S. Chan, S.H. Chung. «Multicriterion genetic optimization for due date assigned distribution network problems.» Decis Support Syst, 39, 4, pp. 661-675 (2005).

14. C.-Y. Chen, Z.-Y. Zhao, M.O. Ball. «Quantity and due date quoting available to promise.» Inform Syst Front, 3, 4, pp. 477-488 (2001).

15. C.-Y. Chen, Z.-Y. Zhao, M.O. Ball. «A model for batch advanced availableto-promise.» Prod Oper Manag, 11, pp. 424-440, (2002).

16. C. Abid, S. D'Amours, B. Montreuil. «Collaborative order management in distributed manufacturing.» Int J Prod Res, 42, 2, pp. 283-302 (2004).

17. W.J. Hopp, M.L.R. Sturgis. «Quoting manufacturing due dates subject to a service level constraint.» IIE Transactions, 32, 9, pp. 771-784, (2000).

18. M.G. Hegedus, W.J. Hopp. «Due date setting with supply constraints in systems using MRP.» Comput Ind Eng, 39, pp. 293-305, (2001)

19. A. Cakravastia, N. Nakamura, «Model for negotiating the price and due date for a single order with multiple suppliers in a make-to-order environment.» Int J Prod Res, 40, 14, pp. 3425-3440, (2002).

20. C. Kilger, C. Schneeweiss, «Demand Fulfilment and ATP.»161-175, Supply Chain Management and Advanced Planning. editado por H. Stadtler, C. Kilger. (Springer) (2002). 
21. A. Hammami, P. Burlat, J.P. Campagne. «Evaluating orders allocation within networks of firms.» Int J Prod Econ, 86, pp. 233-249 (2003).

22. M. Xiong, S.B. Tor, L.P. Khoo, C.-H. Chen. «A web-enhanced dynamic BOM-based available-to-promise system.» Int J Prod Econ, 84, pp. 133-147 (2003).

23. H. Stadtler. «Supply chain management and advanced planning-basics, overview and challenges.» Eur J Oper Res, 163, 3, pp. 575-588 (2005).

24. I. Tortajada, G. Peris-Fajarnés, M. Aguilar, P. Latorre. «Análisis del proceso de clasificación cerámico».Bol. Soc. Esp. Ceram. V., 45 [1], 22-27 (2006).
25. F. Alarcón «Desarrollo de una Arquitectura para la definición del proceso de Comprometer Pedidos en contextos de Redes de Suministro Colaborativas. Aplicación a una Red compuesta por Cadenas de Suministro en los Sectores Cerámico y del Mueble.» Tesis Doctoral, Universidad Politécnica de Valencia (2005)

26. M.M.E. Alemany, A. Ortiz, F.Alarcón, F.-C. Lario, M. A. Bengochea, «El proceso de comprometer pedidos de un paquete de productos integrado por productos del sector cerámico y productos complementarios: Descripción de la metodología de solución», Bol. Soc. Esp. Ceram. V., 46 [1], 29-38 (2007).

Recibido: 23.01.06

Aceptado: 10.04 .06 